\title{
Changes in complete blood count parameters influenced by endocrine disorders
}

\author{
Ewelina Szczepanek-Parulska, Martyna Adamska, Oliwia Korda, Weronika Kosicka, Dagmara \\ Skowrońska, Anna Świejkowska, Dominika Tuzimek, Daniela Dadej, Aleksandra Krygier, Marek Ruchała
}

Department of Endocrinology, Metabolism, and Internal Medicine, Poznan University of Medical Sciences, Poznan, Poland

\begin{abstract}
Complete blood count is one of the most common diagnostic methods used in everyday practice. Hormonal status is known to affect blood count parameters. The aim of this study is to summarize changes in blood count that may indicate endocrine disorders, based on a literature review. Red cell parameters deteriorate in thyroid disorders including autoimmune thyroiditis and tend to resolve with appropriate treatment implementation. The most frequent form of anaemia associated with thyroid dysfunction is normocytic anaemia. Macrocytic anaemia is more typical of autoimmune thyroiditis-induced hypothyroidism, while microcytic anaemia is more common in hyperthyroidism. Unexplained anaemia or an increase in red cell distribution width should prompt the investigation of thyroid disorders. Cushing's disease may manifest as an increase in white blood cells and platelets. In the blood smear, neutrophilia is often present, while lymphocytes and eosinophils may be within the lower normal range. Hypercortisolism may induce both hyperaemia and anaemia. In hypopituitarism, a decrease in red blood cell count, haemoglobin, haematocrit, and platelets is observed. Acromegaly may be accompanied by an increase in mean corpuscular volume of erythrocytes. Testosterone deficiency is manifested by a decrease in red cell parameters, whereas hyperandrogenism may lead to polycythaemia. In polycystic ovary syndrome an increase in white blood cell count reflects an underlying inflammatory state. Complete blood count analysis is an easily available and cost-effective additional tool in the diagnosis and treatment monitoring of endocrine disorders. (Endokrynol Pol 2021; 72 (2): 261-270)
\end{abstract}

Key words: complete blood count; endocrine diseases; hyperthyroidism; hypothyroidism; acromegaly; Cushing's disease

\section{Introduction}

Peripheral blood count is one of the most accessible, cost-effective, and frequently performed diagnostic tests in medical practice. Both venous whole blood sampling and subsequent laboratory analysis are technically simple, allowing the results to be obtained very quickly. Abnormal results may direct further differential diagnosis.

The measured parameters include the following: red blood cells (RBC), haemoglobin (HGB), white blood cells (WBC), platelets (PLT), and haematocrit (HCT). Both manual and automatic blood smears enable quantitative and qualitative assessment of blood cells subpopulations. For leukocytes the proportion of each type in the total pool of white blood cells is determined. The types are as follows: granulocytes - neutrophils, eosinophils and basophils, and agranulocytes: lymphocytes and monocytes. We also receive information on red blood cells parameters - mean corpuscular haemoglobin $(\mathrm{MCH})$, mean corpuscular haemoglobin concentration (MCHC), mean corpuscular volume (MCV) and detailed platelets parameters including MPV - mean platelet volume, PDW - platelet distribution width, which is a platelet volume variability index, $\mathrm{PCT}$ - plateletcrit, and P-LCR - platelet large cell ratio, describing the percentage of large platelets in the total number of thrombocytes.

The hormonal status may influence the parameters assessed in the blood count, thus their changes may reflect endocrine diseases. The aim of this study is to summarise current literature on blood count changes that should prompt diagnosis towards endocrine disorders not only by endocrinologists but by all physicians.

\section{Thyroid disorders}

\section{Hypothyroidism and thyroiditis}

In countries well supplied with iodine, the most common cause of hypothyroidism is chronic autoimmune thyroiditis (Hashimoto's disease), which is included in a wider group of autoimmune thyroid diseases 
(AITDs) [1]. The thyroid hormones stimulate erythropoiesis through increased expression of the erythropoietin (EPO) gene and its production in kidneys [2]. Previous research confirmed that increased triiodothyronine (T3) stimulates a colony of erythrocytes in contrast to hypothyroidism that decreases activity of erythroid cells in the bone marrow and erythropoiesis. In up to $60 \%$ of hypothyroid patients, normocytic anaemia of different aetiology can be observed, while in Hashimoto's thyroiditis a macrocytic anaemia is the most common [1]. It might be explained with accompanying Addison-Biermer anaemia associated with the presence of autoantibodies against the parietal cells or Castle's internal factor, which leads to the vitamin B12 malabsorption. On the other hand, iron deficiency in the course of hypothyroidism further exacerbates anaemia by reducing the synthesis of thyroid hormones and deteriorating the viability of erythrocytes. In microcytic iron deficiency anaemia an increase in in the degree of erythrocytes anisocytosis (red cell distribution width, RDW) is observed. The exact pathomechanism of iron homeostasis disturbances in patients with Hashimoto's thyroiditis is not well elucidated. Proper L-thyroxine replacement therapy normalises haematological parameters [1]. Additional iron substitution in subclinical hypothyroidism increases the effectiveness of L-thyroxine treatment. According to Shakir et al., in patients with anaemia the tolerance of L-thyroxine therapy may be worse [3]. Therefore, it is advised to initiate treatment with iron substitution, then add L-thyroxine. The study by Bremner et al. confirmed that even subtle changes in thyroid function in euthyroid patients may influence the red cell indicators [4]. Hernik et al. observed changes in blood count parameters, especially a gradual reduction of RDW in hypothyroid patients upon restoration of euthyroid state, which reflects improvement of erythropoiesis [1]. In another paper an increased RDW was also observed in the course of hypothyroidism due to Hashimoto's thyroiditis [5]. Interestingly, Aktas et al. suggested that increased RDW may be indicative of Hashimoto's thyroiditis even in euthyroid patients [6]. Additionally, both MCV and $\mathrm{MCH}$ may be lower in hypothyroidism in comparison to euthyroid state [1].

Subacute thyroiditis (SAT) is a specific type of thyroiditis, which comprises hyper- and hypothyroid phases accompanied by essential changes in complete blood count. The following parameters: RDW, HGB, $\mathrm{HCT}, \mathrm{MCV}, \mathrm{MCH}$, and $\mathrm{MCHC}$ were found to be lower in comparison to healthy subjects [7].

Pregnancy is another state predisposing to anaemia. In the $4^{\text {th }}$ week of pregnancy, an increase in plasma volume and number of erythrocytes is observed, but it is proportionally less pronounced than the increase in the volume of plasma. The physiological anaemia in pregnant women improves haemodynamic circulation in the course of increased resistance of blood vessels and increased blood flow through uteroplacental vessels occurring during pregnancy, which provides a better blood supply. Additionally, the level of iron is decreased due to increased utilization and transport to the foetus. On the other hand, iron deficiency is often linked to thyroid diseases, as less iron reduces the activity of thyroid peroxidase - an enzyme essential for the production of thyroid hormones. Concluding, in pregnant women a complete blood count should be monitored, and in case of anaemia, diagnostics directed towards thyroid diseases should be initiated, even if they were not diagnosed before pregnancy [8].

Keskin et al. described differences in haematological parameters between patients with Hashimoto's thyroiditis and a healthy control group, both being euthyroid [9]. They observed higher levels of lymphocytes in comparison to the control group. Additionally, Hashimoto's group presented lower level of HGB and higher MPV but with no difference in WBC. Next, they confirmed a significantly higher neutrophil-lymphocyte ratio (NLR) and platelet-lymphocyte ratio (PLR) in Hashimoto's patients. The research of Erikci et al. demonstrated higher MPV and PDW in subclinical hypothyroidism compared to euthyroidism [10]. The study by Hernik et al. did not demonstrate changes in platelet count in hypothyroid state [1]. In patients suffering from SAT an increased level of platelets and decreased PDW, MPV, and P-LCR were observed. Increased platelet count may result from a decreased level of megakaryocytes that accumulate iron, which leads to increased synthesis of poliploidal megakaryocytes. Thrombocytosis may also be explained by an inflammatory reaction [7]. Kutluturk et al. described the impact of changes in the level of thyroid hormones on platelets in 58 patients with papillary thyroid carcinoma in three phases of treatment: preoperative (euthyroidism), in hypothyroidism before radioiodine treatment (RAI), and subclinical hyperthyroidism after RAI (up to 6 months). They did not notice significant differences in the level of plates, MPV, NLR, or PLR in the hypothyroid phase [11].

\section{Hyperthyroidism}

Hyperthyroidism may have different aetiology, but in countries well supplied with iodine the most common cause is Graves' disease. In 1988, Ford and Carter noticed a significant impact of thyrotoxicosis on all haematopoietic cell lines [12]. In thyrotoxicosis, changes 
of complete blood count depend on the cause. The mechanism of changes is not fully understood.

In a thyrotoxic state, anaemia concerns $10-34 \%$ of all patients with cytopaenia [13]. One of the possible explanations is that excess thyroid hormones increase body metabolism and demand for oxygen [14]. Next, hypoxia stimulates the erythropoietin gene expression and its synthesis in the kidneys [13]. The erythropoietin potentiates erythropoiesis and increases demand for iron, folic acid, and vitamin B12. The most likely theory is a mixed aetiology of anaemia [2]. In hyperthyroidism, the most common type of anaemia is microcytic anaemia, which results from iron deficiency [13, 15]. Parameters that enable the diagnosis include increased RDW and decreased MCV. Normocytic anaemia may occur in Graves' disease with concomitant bone marrow hypoplasia [13]. Macrocytic anaemia occurs due to deficiency of folic acid and vitamin B12 [13, 15], whereas treatment with antithyroid drugs may induce iatrogenic aplastic anaemia [13].

The potential link between anaemia and thyroid diseases may be hepcidin, which is an important regulator of iron homeostasis. According to Krygier et al., the hepcidin concentration decreases during the treatment of Graves' disease [16]. Additionally, they observed an improvement in haematological parameters, especially increase in $\mathrm{MCV}$ and $\mathrm{MCH}$.

Leukopaenia accounts for $15-30 \%$ of all cytopaenia cases, while neutropaenia concerns $5-18 \%$ [13, 17]. The pathomechanism is not well recognized. On one hand, granulopoiesis is limited by a decreased amount of granulocytes in bone marrow. On the other hand, there is a hypothetical immunological mechanism linked to the identification of antibodies directed against neutrophils in patients with thyrotoxicosis [13].

Agranulocytosis is often a result of therapy with anti-thyroid drugs (ATD). According to Japanese researchers, the lower level of granulocytes may occur in up to $70 \%$ patients receiving ATD therapy [18]. Therefore, before introduction of ATD, it is crucial to evaluate complete blood count and monitor this parameter, particularly in the case of fever or throat angina [19].

Thrombocytopaenia accounts for $2-5 \%$ of all cytopaenia cases [13]. Previous studies described the occurrence of antibodies against platelets in patients with thyrotoxicosis. Bagir et al. estimated the significance of MPV to predict the recurrence of Graves' disease; a higher level of MPV was confirmed in the group with recurrence of hyperthyroidism in comparison to the group of patients in remission [20]. The authors explain this phenomenon by an increased metabolic rate due to hyperthyroidism rather than an autoimmune reaction. Turan et al. reported higher level of platelets in patients with untreated Graves' disease [17]. Franchini et al. underlined the correlation between hyperthyroidism and idiopathic thrombocytopaenic purpura [17]. Ford et al. observed no changes in platelet count in hyperthyroidism. They demonstrated a significant decrease in MPV and PCT and slight increase in PDW upon euthyroidism restoration after the phase of hyperthyroidism [12]. Similarly, in the study by Kutluturk et al., MPV, which reflects the pace of plates production and activation, was significantly elevated in subclinical hyperthyroidism compared to euthyroidism [11].

Previously, pancytopaenia in the course of Graves' disease was described occasionally [13]. Initially, pancytopaenia was linked to deficiency of iron, vitamin B12, and coexisting diseases, but in most described cases those disturbances were not observed. Pancytopaenia is generally chronic and well tolerated [13, 22]. Pincet and Gorostidi reported no severe anaemia complications such as intensive bleeding in the course of thrombocytopaenia or infection due to leukopaenia [22]. Next, the parameters of complete blood count returned to normal range after euthyroidism was achieved [13, 14, 23, 24]. For authors the hyperthyroidism was an obvious explanation of the previous unexplained pancytopaenia [22]

A Polish study on 59 children with de novo diagnosed Graves' disease confirmed the previously presented observations. Neutropaenia was noted in $37.3 \%$ of patients, the decreased level of MCV occurred in $32.2 \%$ of children, and $22 \%$ of them had elevated haemoglobin. The decreased levels of RBC, WBC, and PLT were observed in $13.6 \%, 8.5 \%$, and $5 \%$ of patients, respectively. Higher RDW occurred in $15.3 \%$ of the study group. In most cases, the changes in the studied parameters were not significant and severe anaemia, neutropaenia, or thrombocytopaenia were not found. The studies showed statistically significant improvement after ATD therapy. Additionally, no changes in $\mathrm{MCV}, \mathrm{WBC}$, or neutrophils were noticed [25].

\section{Pituitary disorders}

\section{Acromegaly}

Acromegaly is a rare endocrine disorder with two to four new cases per million persons per year and the incidence of 50-70 cases per million inhabitants $[26,27]$. The most common cause of acromegaly is a growth hormone $(\mathrm{GH})$-secreting pituitary adenoma [28]. Changes in the blood count commonly accompany acromegaly [29]. Regular observation of basic blood parameters may be of value considering the high morbidity and mortality due to cardiovascular 
diseases in this group of patients [30]. Current studies have demonstrated alterations in RDW, MCV, and MPV in acromegaly. Uçler et al. compared RDW between patients with active and inactive disease treated either only surgically or with adjuvant somatostatin analogues (SSA) [31]. RDW was significantly higher among patients with inactive acromegaly receiving SSA following surgery. For surgical treatment alone, the results did not reach significance. Strauch et al. observed increased MCV values in male patients with active acromegaly compared to female patients and healthy controls [32]. MCV correlated positively with the GH concentration, suggesting that excessive endogenous $\mathrm{GH}$ secretion is responsible for a reversible increase in MCV in acromegaly.

MPV has aroused interest as a useful indicator of cardiovascular risk [33]. Research on MPV in acromegaly is inconclusive. Several studies have shown an increased MPV in acromegaly compared to healthy individuals [34-36], while another demonstrated no significant differences [37, 38]. Surgical treatment with concomitant use of SSA was found to either increase or decrease MPV, whereas in patients treated only surgically no changes of MPV were noted [31, 37]. The treatment with SSA may thus increase MPV and be associated with greater risk of cardiovascular diseases. Demirpence et al. demonstrated a significant decrease in MPV following surgery without adjuvant therapy [35]. MPV values remained unchanged in the active phase of the disease and in patients in remission who received SSA after surgery. These results suggest that, although acromegaly is pharmacologically controlled with SSAs, the risk of cardiovascular diseases may remain elevated.

Cardiovascular diseases are associated with chronic inflammation. The ratios of neutrophils to leukocytes and platelets to leukocytes are good indicators of inflammation [39]. Üçler et al. demonstrated a positive correlation between the ratio of neutrophils to lymphocytes and insulin-like growth factor 1 (IGF-1), as well as between the ratio of platelets to lymphocytes and IGF-1 [40]. The authors concluded that an inflammatory state due to uncontrolled IGF-1 secretion may increase the risk of mortality and morbidity in the course of acromegaly.

\section{Hypopituitarism}

Hypopituitarism comprises a group of symptoms related to the deficiency of one or more pituitary hormones. Most commonly it results from damage to the pituitary or hypothalamus due to the sellar lesions, autoimmune diseases, or iatrogenic injuries. Less frequently, it is caused by congenital or developmental defects [41].
Valerio et al. investigated the effect of rhGH treatment on erythropoiesis by assessing RBC, HGB, HCT, and MCV in 19 children with isolated GH deficiency (12 cases) and multiple pituitary hormone deficiency (MPHD; 7 cases). During rhGH treatment HGB, HCT, and RBC increased, which indicates the erythropoietic effects of GH in vivo. The authors hypothesised that the deterioration of red cell parameters in hypopituitarism is mainly attributable to GH deficiency. Balancing the other pituitary axes does not fully restore normal erythropoiesis until the GH deficiency has been corrected [42].

In adults a prolonged administration of $\mathrm{rhGH}$ also increased HGB [43]. Haemoglobin concentration peaked at week 120 of the treatment, with mean increase of $0.73 \pm 0.2 \mathrm{mmol} / \mathrm{L}$. The fold of increase was higher in men. HGB concentration changes correlated with IGF-1. The maximum concentration of HGB was observed in the majority of patients with IGF-1 values close to the normal range. Leukocyte and platelet counts remained unchanged throughout the treatment.

Anaemia is a common feature of hypopituitarism. Nishioka and Haraoka investigated the influence of hydrocortisone and/or levothyroxine replacement therapy on HGB concentration in patients with hypopituitarism [44]. HGB concentrations were significantly lower in patients with hypopituitarism in the corticoid, thyroid, somatotropic, and gonadotropic axes compared with healthy controls. The HGB concentration decreased with the disease duration. In some patients, hydrocortisone and levothyroxine supplementation improved HGB concentrations; however, it was not sufficient in all. These results confirm previous observations that additional $\mathrm{rhGH}$ and/or androgen replacement therapy is necessary to obtain further improvement.

Additionally, the study focused on the morphotic elements of blood. The RBC, HCT, and platelets were significantly lower in patients with hypopituitarism compared to the control group. No differences in WBC or reticulocytes were observed.

Beshyah et al. demonstrated that haemostasis in adult patients treated for hypopituitarism is not disturbed [45]. Twenty-one patients with hypopituitarism, treated with thyroxine, hydrocortisone, and sex hormones, participated in the study. All subjects were GH-deficient and had decreased IGF-1 concentrations compared to the control group. Blood count parameters including HGB, WBC, PLT, and HCT did not differ significantly between groups.

\section{Cushing's disease}

ACTH (adrenocorticotropic hormone)-secreting pituitary adenomas are the most common underlying condition of endogenous hypercortisolaemia [46, 47]. 
Numerous blood count abnormalities are associated with Cushing's syndrome, including an increased or decreased RBC and haemoglobin, as well as elevated WBC and PLT.

Possible explanations of erythrocytosis and elevated haemoglobin include decreased erythrophagocytosis caused by glucocorticoid (GCS) excess and stimulation of erythropoietin synthesis or positive regulation of erythropoiesis (e.g. participation in stress erythropoiesis or blood-loss associated erythropoiesis). Erythrocytosis may be reflected as a typical Cushing's syndrome appearance, namely a red, moon-shaped face [48]. Fluctuations in testosterone concentration in hypercortisolism may also affect blood count. Patients suffering from Cushing's syndrome due to GCS excess are likely to have decreased LH and testosterone concentrations. Because testosterone stimulates RBC proliferation (influence stronger than GCS), the blood count may show decreased HCT, RBC, and HGB $[48,49]$. Polycythaemia observed in Cushing's disease occurs more frequently among women due to the less important role of testosterone in the erythropoiesis regulation. As Cushing's disease occurs predominantly in women, polycythaemia is considered a typical symptom [48].

Another blood count abnormality reported in hypercortisolism is a considerable or slight increase in WBC. Most commonly we observe neutrophilia with eosinophils and lymphocytes below or in lower normal range [48]. The mechanisms responsible for leukocytosis include prolonged half-life and postponed neutrophil apoptosis, decreased migration to tissues, and increased inflow of granulocytes into the bloodstream induced by stress factors such as cortisol or catecholamines [50].

Platelet concentration is either normal or increased [50]. An increased risk of venous thrombo-embolic disease must be considered [51].

\section{Hyperprolactinaemia}

The largest proportion of pituitary adenomas are prolactinomas. They are considered the main cause of hyperprolactinaemia, which is the most common hormonal abnormality among the hypothalamic and pituitary disorders [52, 53].

The most frequent blood count abnormality observed in hyperprolactinaemia is thrombocytosis [54], although the research on this topic is limited. The change of platelet parameters is not observed in the pregnancy-induced hyperprolactinaemia [54]. On the other hand, changes in haemostasis parameters are observed more frequently and include, e.g., increased platelet activation and an increased fibrinogen concentration, which, taken together, contribute to the increased risk of thrombosis, atherosclerosis, and heart disease [55, 21]. This also applies to patients with macroprolactinaemia [55]. Hyperprolactinaemia does not affect total white blood cell counts or other blood count parameters [56].

\section{Adrenal disorders}

\section{Adrenal insufficiency}

In Addison's disease (primary adrenal insufficiency), the most common abnormality regarding blood count parameters is eosinophilia. Eosinophilia in Addison's disease was described in early works from the 1970s [57]. Eosinophil concentration above $500 / \mathrm{mm}^{3}$ was found in 4 out of 26 subjects. In one 21-year-old patient the concentration reached $800 / \mathrm{mm}^{3}$ in the absence of any parasitic infection. Oral administration of cortisol reduced eosinophilia in this patient, while daily use reduced the myeloid production of eosinophils and restored eosinophil concentration. Eosinophilia has also been described in a patient with adrenal insufficiency due to tuberculosis [58]. However, it cannot be clearly stated that eosinophilia is caused solely by low plasma cortisol levels, because it is not present in all patients with Addison's disease.

In patients with adrenal insufficiency, treatment with dehydroepiandrostenedione sulphate (DHEAS) has an immunomodulatory effect, increasing the number of $\mathrm{T}$ cells by stimulating their proliferation. A 12-week exposure of cultured lymphocytes to DHEA$S$ increased their number by more than 10 times while reducing the number of NK cells by $37-43 \%$. Additionally, in one trial, the treatment increased the number of $B$ cells and monocytes [59]. However, an experimental study in mice showed that long-term use of DHEA-S in these animals did not affect the T-cell subpopulation or the production of antibodies after erythrocyte immunization [60].

Addison's disease may coexist with other autoimmune disorders as a part of the autoimmune polyglandular syndrome [61]. Then it may be accompanied by pernicious anaemia - a macrocytic anaemia due to vitamin B12 deficiency.

\section{Pheochromocytoma and hyperaldosteronism}

Blood count changes are also reported in pheochromocytoma - a tumour originating from the adrenal medulla. Experimental studies have shown that high-dose catecholamine administration increases WBC and PLT, and promotes platelet activation, which is also observed in chronic stress [62,63]. Neutrophilia was reported in a patient with phaeochromocytoma and non-cardiac pulmonary oedema [64]. The authors hypothesised that elevated WBC may lead to respiratory failure by increasing the permeability of pulmonary vessels and promoting the development of that complication [64]. 
Zelink et al. compared blood count parameters between patients with pheochromocytoma, primary hyperaldosteronism, and idiopathic arterial hypertension [65]. WBC in pheochromocytoma was significantly higher compared to the group of patients with primary aldosteronism or the control group. On the other hand, the number of platelets was higher in patients with phaeochromocytoma compared to primary hyperaldosteronism, but lower than in the control group. There were no significant differences in RBC and HGB among the studied subgroups. After surgical treatment and normalization of catecholamine concentrations, WBC, neutrophils, and platelets decreased. The influence of catecholamines on the blood morphotic elements is explained by their direct interaction with cell surface receptors: PLT - alpha receptors stimulated by epinephrine, and WBC - adrenoreceptors.

An elevated HGB concentration has also been described in a 20-year-old patient with late diagnosis of Bartter's syndrome type IV. Other morphological parameters (leukocytes, platelets), as well as plasma iron, transferrin, and erythropoietin concentration were within the normal ranges [66]. The reasons for this phenomenon remain unclear. A similar clinical situation, described by Erkelens many years ago, was interpreted by the author with the hypertrophy of the glomerular apparatus, which led to the overproduction of renin and an increase in the synthesis of erythropoietin [67]. It is now known that the glomerular apparatus is not the main site of erythropoietin synthesis, the concentration of which in the described case was within the normal range. The occurrence of erythrocytosis may be the result of polyuria, which, however, also did not apply to this patient.

\section{Premature adrenarche}

Adrenarche is the process of adolescence in which the child's adrenal cortex begins secreting androgen precursors. These mainly include dehydroepiandrosterone and androstenedione. This phenomenon is considered premature when it occurs before the age of 8 years in girls and before the age of 9 years in boys. Adrenal hormones promote secondary sex characteristics such as the appearance of pubic and underarm hair, acne, and sweat with a characteristic smell. When accompanied by gonadarche, it is classified as premature puberty. Adrenarche praecox (AP) is more common in girls - 9/10 patients [68]. Reports indicate a relationship between $\mathrm{AP}$ and altered values of blood count parameters in patients compared to the group of healthy people. It has been shown that the RBC level is higher in girls with AP than in the same sex in the control group. Such a relationship did not occur in the group of boys. At the same time, the remaining blood count parameters did not differ significantly between the affected and healthy subjects of both sexes [68].

\section{Disorders of the secretory function of the gonads}

\section{Polycystic ovary syndrome}

Polycystic ovary syndrome (PCOS) is an endocrine-metabolic disorder that affects 5 to $25 \%$ of women in reproductive age [69-72]. Characteristic features for this disease include oligo- or anovulation, polycystic ovaries on ultrasound imaging, and clinical and biochemical hyperandrogenism. PCOS is also associated with metabolic disorders such as glucose intolerance, insulin resistance, and obesity. In patients with PCOS, an increase in many markers of inflammation is also observed [72]. Chronic inflammation is a significant risk factor for the development of atherosclerosis and cardiovascular diseases.

WBC is an indicator of inflammation and is routinely measured in the blood count. In PCOS patients a significantly higher WBC was observed compared to healthy women, matched for age and BMI. Increased concentration of leukocytes is an independent predictor of inflammation and atherosclerosis [72]. Research indicates a correlation between WBC, CRP, insulin resistance, BMI, and visceral fat. However, it is still not fully understood whether inflammation is directly related to PCOS or reflects the insulin resistance and obesity associated with the syndrome [72]. Other morphological parameters considered as inflammation indicators include PLT, MPV, PDW, and PCT. Cardiovascular, cerebrovascular, and inflammatory diseases influence PLT counts. MPV, PLR and NLR indices have also been considered as markers of inflammation. However, studies in PCOS patients demonstrated no changes in the above-mentioned blood count parameters, and thus they are not applicable in this syndrome [72]. Due to the limited research in this field, further studies are necessary to confirm those findings. Metformin therapy in PCOS reduces WBC (including lymphocyte and monocyte count) and thereby reduces inflammation and the risk of atherosclerosis and cardiovascular diseases [69].

\section{Male hypogonadism}

Male hypogonadism results in gonadal dysfunction. Due to its aetiology, hypogonadism is classified as:

- hypergonadotropic (primary) hypogonadism, in which the testes are unable to perform an endocrine or reproductive function due to damage to their structure. This group includes among others: gonadal dysgenesis, congenital lack of testes, Klinefelter syndrome; 
- hypogonadotropic (secondary) hypogonadism, resulting from hypothalamic-pituitary insufficiency and decreased gonadotrophins. It occurs in the course of hyperprolactinaemia, Kallmann's syndrome, hypopituitarism, isolated FSH deficiency or might be idiopathic;

- mixed hypogonadism - late-onset hypogonadism $(\mathrm{LOH})$

- syndromes associated with the target tissue resistance to sex hormones - aromatase deficiency, androgen insensitivity syndrome [73].

Clinical presentation of male hypogonadism results from testosterone deficiency. Erythropoiesis is an androgen-dependent process. Therefore, a mild anaemia is common in hypogonadal patients. Substitution of testosterone restores proper erythropoiesis and might induce polycythaemia in up to $25 \%$ of patients [73-76]. Blood count is one of the basic tests allowing indirect assessment of the effectiveness of the treatment of hypogonadism. Regular blood count monitoring, with particular attention paid to $\mathrm{HCT}$, is recommended during testosterone therapy $[73,74$, 76]. If HCT increases above $54 \%$, the dose should be reduced or the intramuscular drug administration route changed to transdermal [74]. In some cases, a phlebotomy is recommended. If the aforementioned actions are unsuccessful in decreasing HCT, testosterone therapy should be discontinued and restarted after normalization of this parameter.

In idiopathic hypogonadotropic hypogonadism (IHH) HGB, HCT, and MCV were reported to decrease compared to the healthy individuals. Conversely, RDW in patients with $\mathrm{IHH}$ was higher than in healthy people, which reflects a greater anisocytosis of red blood cells. In IHH PLT increased significantly compared to the control group. The study cited above concerned newly diagnosed, untreated $\mathrm{IHH}$ patients, thus changes in the erythropoiesis are a direct consequence of this disease [75].

\section{Doping}

Doping involves the use of methods aimed at increasing the physical performance of athletes, which at the same time go beyond the limits of conventional training. Due to gradual blurring of boundaries between "proper" training and doping, the latter can be considered as methods that require medical intervention.

According to the 2015 World Anti-Doping Agency (WADA) definition, doping is a breach of at least one of the anti-doping rules included in the Anti-Doping Code. These include: the presence of a Prohibited Substance, its metabolites or markers in a sample collected from an Athlete, use / attempted use by an Athlete of a prohibited substance or method, and evasion or re- fusal to collect samples for doping testing. The code also lists the following: possession of prohibited substances, trade or attempted trade in such substances, as well as administering or attempting to administer them to an athlete [77].

In order to detect doping and disqualify athletes using illegal methods, WADA has developed the Athlete Biological Passport (ABP). It includes, inter alia, haematological parameters. Peripheral blood counts include parameters listed in the haematological $\mathrm{ABP}$ : HCT, HGB, MCH, MCV, MCHC, PLT, RBC, WBC, and RET [63]. In view of the above, an endocrinologist can assess certain abnormalities characteristic for doping on the basis of morphology.

Androgenic anabolic steroids (AAS) are a widely used method of doping, both in professional athletes and amateurs, being synthetic testosterone derivatives. They are mainly applied to increase the muscle mass; therefore, their use is often associated with sports that require significant muscle strength in a short time, e.g. weight lifting or sprinting. Smaller doses of anabolic steroids are taken by cyclists to increase the erythrocyte count and haematocrit, which increases the supply of oxygen to the cells [78].

Chrostowski et al. investigated the effect of high-dose ASS administration on the renin-angiotensin-aldosterone axis. Forty bodybuilders were enrolled and divided into ASS-urine positive and negative groups. In individuals using AAS, significantly higher levels of RBC, HGB, and HCT were reported compared to the group not taking ASS [79]. These results confirm both the effectiveness of AAS as a doping agent and their influence on blood count parameters. Properly performed differential diagnosis should include doping as one of the potential causes of abnormally high blood count parameters.

\section{Conclusions}

In the course of thyroid dysfunction and AITD, the worsening of RBC parameters is observed, which improve following successful therapy. Agranulocytosis may be one of side effects of ATD used for hyperthyroidism therapy. The most common form of anaemia in the case of thyroid dysfunction is normocytic anaemia. Macrocytic anaemia is more typical of hypothyroidism, while microcytic is more typical for hyperthyroidism. Unexplained anaemia or an increase in RDW should prompt the diagnostics directed towards thyroid diseases. In the course of Cushing's disease an increase in PLT and WBC is observed. The most common is neutrophilia, while the concentration of eosinophils and lymphocytes may be in the lower reference ranges. In hypercortisolism, both anaemia and hyperaemia may 
occur. In hypopituitarism, a decrease in RBC, HGB, $\mathrm{HCT}$, and PLT is observed. In patients with acromegaly, an increase in the MCV parameter is detected. Testosterone deficiency is accompanied by a decrease in RBC parameters, while androgen excess may induce polycythaemia. Routine control of blood count is recommended during the therapy with testosterone. In women with PCOS features of chronic inflammatory state are detected, which may be reflected by an increase in WBC. Evaluation of complete blood count is a useful tool in the basic diagnostics and monitoring of endocrine disorders.

\section{Conflict of interest}

Authors declare there is no conflict of interest.

\section{Funding}

The manuscript received no specific funding.

\section{References}

1. Hernik A, Szczepanek-Parulska E, Filipowicz D, et al. The hepcidin concentration decreases in hypothyroid patients with Hashimoto's thyroiditis following restoration of euthyroidism. Sci Rep. 2019; 9(1): 16222, doi: 10.1038/s41598-019-52715-3, indexed in Pubmed: 31700042.

2. Szczepanek-Parulska E, Hernik A, Ruchała M. Anemia in thyroid diseases. Pol Arch Intern Med. 2017; 127(5): 352-360, doi: 10.20452/pamw.3985, indexed in Pubmed: 28400547.

3. Shakir KM, Turton D, Aprill BS, et al. Anemia: a cause of intolerance to thyroxine sodium. Mayo Clin Proc. 2000; 75(2): 189-192, indexed in Pubmed: 10683660.

4. Bremner AP, Feddema P, Joske DJ, et al. Significant association between thyroid hormones and erythrocyte indices in euthyroid subjects. Clin Endocrinol (Oxf). 2012; 76(2): 304-311, doi: 10.1111/j.1365-2265.2011.04 228.x, indexed in Pubmed: 21913954.

5. Montagnana M, Lippi G, Targher G, et al. The red blood cell distribution width is associated with serum levels of thyroid stimulating hormone in the general population. Int J Lab Hematol. 2009; 31: 581-582, doi: 10.111 1/j.1751-553X.2008.01082.x, indexed in Pubmed: 18616753.

6. Aktas G, Sit M, Dikbas O, et al. Could red cell distribution width be a marker in Hashimoto's thyroiditis? Exp Clin Endocrinol Diabetes. 2014; 122: 572-574, doi: 10.1055/s-0034-1383564, indexed in Pubmed: 25380549.

7. Hernik A, Szczepanek-Parulska E, Filipowicz D, et al. Hepcidin and Iron Homeostasis in Patients with Subacute Thyroiditis and Healthy Subjects. Mediators Inflamm. 2019; 2019: 5764061, doi: 10.1155/2019/5764061, indexed in Pubmed: 30936776.

8. He L, Shen C, Zhang Y, et al. Evaluation of serum ferritin and thyroid function in the second trimester of pregnancy. Endocr J. 2018; 65(1): 75-82, doi: 10.1507/endocrj.EJ17-0253, indexed in Pubmed: 29033409.

9. Keskin H, Kaya Y, Cadirci K, et al. Elevated neutrophil-lymphocyte ratio in patients with euthyroid chronic autoimmune thyreotidis. Endocr Regul. 2016; 50(3): 148-153, doi: 10.1515/enr-2016-0017, indexed in Pubmed: 27560798.

10. Erikci AA, Karagoz B, Ozturk A, et al. The effect of subclinical hypothyroidism on platelet parameters. Hematology. 2009; 14(2): 115-117, doi: 10.1179/102453309X385124, indexed in Pubmed: 19298725.

11. Kutluturk F, Gul SS, Sahin S, et al. Comparison of Mean Platelet Volume, Platelet Count, Neutrophil/Lymphocyte Ratio and Platelet/Lymphocyte Ratio in the Euthyroid, Overt Hypothyroid and Subclinical Hyperthyroid Phases of Papillary Thyroid Carcinoma. Endocr Metab Immune Disord Drug Targets. 2019; 19(6): 859-865, doi: 10.2174/18715303196661 90206125545, indexed in Pubmed: 30727930.

12. Ford HC, Carter JM. The haematology of hyperthyroidism: abnormalities of erythrocytes, leucocytes, thrombocytes and haemostasis. Postgrad Med J. 1988; 64(756): 735-742, doi: 10.1136/pgmj.64.756.735, indexed in Pubmed: 3076660

13. Garla VV, Abdul Salim S, Yanes-Cardozo LL. Pancytopenia: a rare complication of Graves' disease. BMJ Case Rep. 2018; 2018, doi: 10.1136/bcr-2017-223887, indexed in Pubmed: 29525760.

14. Naji P, Kumar G, Dewani S, et al. Graves' disease causing pancytopenia and autoimmune hemolytic anemia at different time intervals: a case report and a review of the literature. Case Rep Med. 2013; 2013: 194542, doi: 10.1155/2013/194542, indexed in Pubmed: 24319463.

15. Dorgalaleh A, Mahmoodi M, Varmaghani B, et al. Effect of thyroid dysfunctions on blood cell count and red blood cell indice. Iran J Ped Hematol Oncol. 2013; 3: 73-77, indexed in Pubmed: 24575274.

16. Krygier A, Szczepanek-Parulska E, Filipowicz D, et al. Changes in serum hepcidin according to thyrometabolic status in patients with Graves disease. Endocr Connect. 2020; 9(3): 234-242, doi: 10.1530/ec-20-0017, indexed in Pubmed: 32069222.

17. Turan E. Evaluation of neutrophil-to-lymphocyte ratio and hematologic parameters in patients with Graves' disease. Bratisl Lek Listy. 2019; 120(6): 476-480, doi: 10.4149/BLL_2019_076, indexed in Pubmed: 31223030.

18. Nakamura H, Miyauchi A, Miyawaki N, et al. Analysis of 754 cases of antithyroid drug-induced agranulocytosis over 30 years in Japan. J Clin Endocrinol Metab. 2013; 98(12): 4776-4783, doi: 10.1210/jc.2013-2569, indexed in Pubmed: 24057289.

19. Ross DS, Burch HB, Cooper DS, et al. 2016 American Thyroid Association Guidelines for Diagnosis and Management of Hyperthyroidism and Other Causes of Thyrotoxicosis. Thyroid. 2016; 26(10): 1343-1421, doi: 10.1089/thy.2016.0229, indexed in Pubmed: 27521067.

20. Bagir GS, Haydardedeoglu FE, Bakiner OS, et al. Mean Platelet Volume in Graves' disease: A Sign of Hypermetabolism Rather than Autoimmunity? Pak J Med Sci. 2017; 33(4): 871-875, doi: 10.12669/pjms.334.12659, indexed in Pubmed: 29067056

21. Franchini M, Lippi G, Manzato F, et al. Hemostatic abnormalities in endocrine and metabolic disorders. Eur J Endocrinol. 2010; 162(3): 439-451, doi: 10.1530/EJE-09-0958, indexed in Pubmed: 19934268.

22. Pincet L, Gorostidi F. Graves Disease Causing Pancytopenia: Case Report and Literature Review. Clin Med Insights Case Rep. 2018; 11: 1179547618781090, doi: 10.1177/1179547618781090, indexed in Pubmed: 30083058.

23. Rafhati AN, See CK, Hoo FK, et al. A report of three cases of untreated Graves' disease associated with pancytopenia in Malaysia. Electron Physician. 2014; 6(3): 877-882, doi: 10.14661/2014.877-882, indexed in Pubmed: 25763162.

24. Baagar KA, Siddique MA, Arroub SA, et al. Atypical Complications of Graves' Disease: A Case Report and Literature Review. Case Rep Endocrinol. 2017; 2017: 6087135, doi: 10.1155/2017/6087135, indexed in Pubmed: 28348902.

25. Artemniak-Wojtowicz D, Witkowska-Sedek E, Borowiec A et al. Peripheral blood picture and aminotransferase activity in children with newly diagnosed Graves' disease at baseline and after the initiation of antithyroid drug therapy. Cent Eur J Immunol. 2019; 44(2): 132-137, doi: 10.5114/ceji.2019.87063, indexed in Pubmed: 31530982.

26. Bolanowski M, Ruchała M, Zgliczyński W, et al. Diagnostics and treatment of acromegaly - updated recommendations of the Polish Society of Endocrinology. Endokrynol Pol. 2019; 70(1): 2-18, doi: 10.5603/EP.a2018.0093, indexed in Pubmed: 30843181.

27. Giustina A, Barkan A, Beckers A, et al. A Consensus on the Diagnosis and Treatment of Acromegaly Comorbidities: An Update. J Clin Endocrinol Metab. 2020; 105(4), doi: 10.1210/clinem/dgz096, indexed in Pubmed: 31606735.

28. Bolanowski M, Ruchała M, Zgliczyński W, et al. Acromegaly--a novel view of the patient. Polish proposals for diagnostic and therapeutic procedures in the light of recent reports. Endokrynol Pol. 2014; 65(4): 326-331, doi: 10.5603/EP.2014.0045, indexed in Pubmed: 25185857.

29. Mazziotti G, Marzullo P, Doga M, et al. Growth hormone deficiency in treated acromegaly. Trends Endocrinol Metab. 2015; 26(1): 11-21, doi: 10.1016/j.tem.2014.10.005, indexed in Pubmed: 25434492.

30. Colao A, Grasso LFS, Di Somma C, et al. Acromegaly and Heart Failure. Heart Fail Clin. 2019; 15(3): 399-408, doi: 10.1016/j.hfc.2019.03.001, indexed in Pubmed: 31079698.

31. Ucler R, Aslan M, Atmaca M, et al. The effect of disease control on mean platelet volume and red blood cell distribution in patients with acromegaly. Int J Clin Exp Med. 2015; 8: 6060-6066, indexed in Pubmed: 26131204.

32. Strauch G, Lego A, Therain F, et al. Reversible plasma and red blood cells volumes increases in acromegaly. Acta Endocrinol (Copenh. 1997; 85: 465-478, doi: 10.1530/acta.0.0850465, indexed in Pubmed: 577334

33. Vizioli L, Muscari S, Muscari A. The relationship of mean platelet volume with the risk and prognosis of cardiovascular diseases. Int J Clin Pract. 2009; 63(10): 1509-1515, doi: 10.1111/j.1742-1241.2009.02070.x, indexed in Pubmed: 19769707.

34. Arpaci D, Kuzu F, Unal M, et al. Assessment of Mean Platelet Volume and its Effect on Disease Control in Patients with Acromegaly. Clin Lab. 2016; 62(11): 2167-2171, doi: 10.7754/Clin.Lab.2016.160404, indexed in Pubmed: 28164675.

35. Demirpence M, Yasar HY, Colak A, et al. Mean Platelet Volume and Platelet Function Analysis in Acromegalic Patients before and after Treatment. Acta Endocrinol (Buchar). 2016; 12(4): 401-406, doi: 10.4183/aeb.2016.401, indexed in Pubmed: 31149122. 
36. Unübol M, Güney E, Türe $\mathrm{M}$, et al. Mean platelet volume and arterial stiffness in patients with acromegaly. Anadolu Kardiyol Derg. 2014; 14(5): 456-463, doi: 10.5152/akd.2014.4898, indexed in Pubmed: 24901024

37. Ersoy R, Gul K, Solaroglu N, et al. Effect of a six-month treatment with octreotide long acting repeatable (LAR) on mean platelet volume in patients with acromegaly. Endocrine Abstr. 2008; 16.

38. Durmaz S, Carlioglu A, Ayhan E, et al. The effects of octreotide acetate long-acting repeatable on mean platelet volume in acromegaly: octreotidelar may have a detrimental effect on MPV, a new indicato of atherosclerosis. Endocrine Abstr. 2014; 35: 855-855, doi: 10.1530/endoabs.35.p855.

39. Gupta P, Dutta P. Co-Occurrence of Acromegaly and Hematological Disorders: A Myth or Common Pathogenic Mechanism. Integr Med Int. 2017; 4(1-2): 94-100, doi: 10.1159/000478932

40. Üçler R, Aslan M, Atmaca M, et al. Evaluation of blood neutrophil to lymphocyte and platelet to lymphocyte ratios according to plasma glucose status and serum insulin-like growth factor 1 levels in patients with acromegaly. Hum Exp Toxicol. 2016; 35(6): 608-612, doi: 10.1177/0960327115597313, indexed in Pubmed: 26224042.

41. Kluczyński Ł, Gilis-Januszewska A, Rogoziński D, et al. Hypophysitis - new insights into diagnosis and treatment. Endokrynol Pol 2019; 70(3): 260-269, doi: 10.5603/EP.a2019.0015, indexed in Pubmed: 31290557.

42. Valerio G, Di Ma, Salerno M, et al. Assessment of red blood cell indices in growth-hormone-treated children. Horm Res. 1997; 47: 62-66 doi: $10.1159 / 000185433$

43. Ten Have SM, van der Lely AJ, Lamberts SW. Increase in haemoglobin concentrations in growth hormone deficient adults during human recombinant growth hormone replacement therapy. Clin Endocrinol (Oxf). 1997; 47(5): 565-570, doi: 10.1046/j.1365-2265.1997.3241124.x indexed in Pubmed: 9425396.

44. Nishioka H, Haraoka J. Hypopituitarism and anemia: effect of replacement therapy with hydrocortisone and/or levothyroxine. J Endocrinol Invest. 2005; 28(6): 528-533, doi: 10.1007/BF03347241, indexed in Pubmed: 16117194 .

45. Beshyah SA, Markussis V, Harbourne T, et al. Haemostatic mechanisms are normal despite increased vascular mortality in hypopituitary adults. Horm Metab Res. 1993; 25(8): 449-450, doi: 10.1055/s-2007-1002144, indexed in Pubmed: 8225194

46. Łebek-Szatańska A, Stelmachowska-Banaś M, Zieliński G, et al. Corticotropinoma as the underlying cause of intermittent Cushing's syndrome in a patient previously diagnosed with primary pigmented nodular adrenocortical disease. Endokrynol Pol. 2020; 71(3): 273-274, doi: 10.5603/EPa2020.0015, indexed in Pubmed: 32293705.

47. Szczepanek-Parulska E, Cyranska-Chyrek E, Nowaczyk M, et al. Diagnostic Difficulties In a Young Women With Symptoms of Cushing Syndrome. Endocr Pract. 2018; 24(8): 766, doi: 10.4158/EP-2017-0257, indexed in Pubmed: 29498909.

48. Ambrogio AG, De Martin M, Ascoli P, et al. Gender-dependent changes in haematological parameters in patients with Cushing's disease before and after remission. Eur J Endocrinol. 2014; 170(3): 393-400, doi: 10.1530/EJE-13-0824, indexed in Pubmed: 24362410.

49. Ellegala DB, Alden TD, Couture DE, et al. Anemia, testosterone, and pituitary adenoma in men. J Neurosurg. 2003; 98(5): 974-977, doi: 10.3171/jns.2003.98.5.0974, indexed in Pubmed: 12744356

50. Masri-Iraqi H, Robenshtok E, Tzvetov G, et al. Elevated white blood cell counts in Cushing's disease: association with hypercortisolism. Pituitary. 2014; 17(5): 436-440, doi: 10.1007/s11102-013-0522-0, indexed in Pubmed: 24078318.

51. Wagner J, Langlois F, Lim DS, et al. Hypercoagulability and Risk of Venous Thromboembolic Events in Endogenous Cushing's Syndrome: A Systematic Meta-Analysis. Front Endocrinol (Lausanne) 2018; 9: 805, doi: 10.3389/fendo.2018.00805, indexed in Pubmed: 30745894 .

52. Melmed S, Casanueva FF, Hoffman AR, et al. Endocrine Society. Diagnosis and treatment of hyperprolactinemia: an Endocrine Society clinical practice guideline. J Clin Endocrinol Metab. 2011; 96(2): 273-288, doi: 10.1210/jc.2010-1692, indexed in Pubmed: 21296991.

53. Szczepanek-Parulska E, Filipowicz D, Kuśmierek A, et al. Remarkable remission of an invasive giant prolactinoma under high-dose bromocriptine monotherapy. Pol Arch Intern Med. 2017; 127(7-8): 559-560 doi: 10.20452/pamw.4079, indexed in Pubmed: 28817546.

54. Erem C, Kocak M, Nuhoglu I, et al. Blood coagulation, fibrinolysis and lipid profile in patients with prolactinoma. Clin Endocrinol (Oxf). 2010; 73(4): 502-507, doi: 10.1111/j.1365-2265.2009.03752.x, indexed in Pubmed: 20039901.

55. Anaforoglu I, Ertorer ME, Kozanoglu I, et al. Macroprolactinemia, like hyperprolactinemia, may promote platelet activation. Endocrine. 2010; 37(2): 294-300, doi: 10.1007/s12020-009-9304-x, indexed in Pubmed: 20960266.
56. Gerli R, Rambotti P, Nicoletti I, et al. Reduced number of natural killer cells in patients with pathological hyperprolactinemia. Clin Exp Immunol. 1986; 64(2): 399-406, indexed in Pubmed: 3091304.

57. Spry C. Eosinophilia in Addison's disease. Yale J Biol Med. 1976; 49(4): 411-413, indexed in Pubmed: 186962

58. Malu AO, Sanusi BR, Obineche EN. Addison's disease presenting with marked eosinophilia and psychosis. Trop Geogr Med. 1988; 40(3): 241-243, indexed in Pubmed: 3188216.

59. Coles AJ, Thompson S, Cox AL, et al. Dehydroepiandrosterone replacement in patients with Addison's disease has a bimodal effect on regulatory (CD4+CD25hi and CD4+FoxP3+) T cells. Eur J Immunol. 2005; 35(12): 3694-3703, doi: 10.1002/eji.200526128, indexed in Pubmed: 16252254.

60. Miller RA, Chrisp C. Lifelong treatment with oral DHEA sulfate does not preserve immune function, prevent disease, or improve survival in genetically heterogeneous mice. J Am Geriatr Soc. 1999; 47(8): 960-966, doi: 10.1111/j.1532-5415.1999.tb01291.x, indexed in Pubmed: 10443857.

61. Vrkljan AM, Pašalić A Strinović M, et al. Coexistence of Addison's Disease and Pernicious Anemia: Is the New Classification of Autoimmune Polyglandular Syndrome Appropriate? Acta Clin Croat. 2015; 54(2): 232-235, indexed in Pubmed: 26415323.

62. Benschop RJ, Rodriguez-Feuerhahn M, Schedlowski M. Catecholamine-induced leukocytosis: early observations, current research, and future directions. Brain Behav Immun. 1996; 10(2): 77-91, doi: 10.1006/brbi.1996.0009, indexed in Pubmed: 8811932.

63. Larsson PT, Wallén NH, Hjemdahl P. Norepinephrine-induced human platelet activation in vivo is only partly counteracted by aspirin. Circulation. 1994; 89(5): 1951-1957, doi: 10.1161/01.cir.89.5.1951, indexed in Pubmed: 8181117.

64. Sukoh N, Hizawa N, Yamamoto H, et al. Increased neutrophils in bronchoalveolar lavage fluids from a patient with pulmonary edema associated with pheochromocytoma. Intern Med. 2004; 43(12): 1194-1197, doi: 10.2169/internalmedicine.43.1194, indexed in Pubmed: 15645658.

65. Zelinka T, Petrák O, Strauch B, et al. Elevated inflammation marker in pheochromocytoma compared to other forms of hypertension. Neuroimmunomodulation. 2007; 14(1): 57-64, doi: 10.1159/000107289, indexed in Pubmed: 17700041.

66. Heilberg IP, Tótoli C, Calado JT Adult presentation of Bartter syndrome type IV with erythrocytosis. Einstein (Sao Paulo). 2015; 13(4): 604-606, doi: 10.1590/S1679-45082015RC3013, indexed in Pubmed: 26537508.

67. Erkelens DW, Statius van Eps LW. Bartter's syndrome and erythrocytosis. Am J Med. 1973; 55(5): 711-719, doi: 10.1016/0002-9343(73)90196-4 indexed in Pubmed: 4356102.

68. Novello L, Speiser PW. Premature Adrenarche. Pediatr Ann. 2018 47(1): e7-ee11, doi: 10.3928/19382359-20171214-04, indexed in Pubmed: 29323690

69. Orio F, Manguso F, Di Biase S, et al. Metformin administration improves leukocyte count in women with polycystic ovary syndrome: a 6-month prospective study. Eur J Endocrinol. 2007; 157(1): 69-73, doi: 10.1530/EJE-07-0133, indexed in Pubmed: 17609404

70. Dutkowska A, Konieczna A, Breska-Kruszewska J, et al. [Recomendations on non-pharmacological interventions in women with PCOS to reduce body weight and improve metabolic disorders [Zalecenia dotyczące postępowania niefarmakologicznego u kobiet z PCOS celem zmniejszenia masy ciała i poprawy zaburzeń metabolicznych]]. Endokrynol Pol. 2019; 70(2): 198-212, doi: 10.5603/EP.a2019.0006, indexed in Pubmed: 31039273.

71. Milewicz A, Kudła M, Spaczyński RZ, et al. The polycystic ovary syndrome: a position statement from the Polish Society of Endocrinology, the Polish Society of Gynaecologists and Obstetricians, and the Polish Society of Gynaecological Endocrinology. Endokrynol Pol. 2018; 69(4), doi: 10.5603/EP.2018.0046, indexed in Pubmed: 30209800.

72. Rudnicka E, Kunicki M, Suchta K, et al. Inflammatory Markers in Women with Polycystic Ovary Syndrome. Biomed Res Int. 2020; 2020: 4092470, doi: 10.1155/2020/4092470, indexed in Pubmed: 32219132.

73. Nieschlag E. Late-onset hypogonadism: a concept comes of age Andrology. 2020; 8(6): 1506-1511, doi: 10.1111/andr.12719, indexed in Pubmed: 31639279

74. Lunenfeld B, Mskhalaya G, Zitzmann M, et al. Recommendations on the diagnosis, treatment and monitoring of late-onset hypogonadism in men - a suggested update. Aging Male. 2013; 16(4): 143-150, doi: 10 3109/13685538.2013.853731, indexed in Pubmed: 24188520.

75. Pekkolay Z. Idiopathic hypogonadotropic hypogonadism: erythrocyte indices in naive male patients. Eur J Med Invest. 2018, doi: 10.14744/ejmi.2018.19483.

76. Namiki M, Akaza H, Shimazui T, et al. Working Committee on Clinica Practice Guidelines for Late-onset Hypogonadism, Japanese Urological Association/Japanese Society for Study of Aging Male. Clinica practice manual for late-onset hypogonadism syndrome. Int J Urol. 2008; 15(5): 377-388, doi: 10.1111/j.1442-2042.2008.02010.x, indexed in Pubmed: 18452452 
77. Hughes D. The World Anti-Doping Code in sport: Update for 2015. Aust Prescr. 2015; 38(5): 167-170, doi: 10.18773/austprescr.2015.059, indexed in Pubmed: 26648655

78. Bird SR, Goebel C, Burke LM, et al. Doping in sport and exercise: anabolic, ergogenic, health and clinical issues. Ann Clin Biochem. 2016;
53(Pt 2): 196-221, doi: 10.1177/0004563215609952, indexed in Pubmed: 26384361.

79. Chrostowski K, Kwiatkowska D, Pokrywka A, et al. Renin-angiotensin-aldosterone system in bodybuilders using supraphysiological doses of anabolic-androgenic steroids. Biol Sport. 2011; 28(1): 11-17, doi: 10.5604/935862. 